\title{
SUPERSOLUBLE GROUPS OF AUTOMORPHISMS OF COMPACT RIEMANN SURFACES
}

\author{
by GRZEGORZ GROMADZKI and COLIN MACLACHLAN
}

(Received 12 July, 1988)

1. Introduction. Given an integer $g \geqslant 2$ and a class $\mathscr{F}$ of finite groups let $N(g, \mathscr{F})$ denote the order of the largest group in $\mathscr{F}$ that a compact Riemann surface of genus $g$ admits as a group of automorphisms. For the classes of all finite groups, cyclic groups, abelian groups, nilpotent groups, $p$-groups (given $p$ ), soluble groups and finally for metabelian groups, an upper bound for $N(g, \mathscr{F})$ as well as infinite sequences for $g$ for which this bound is attained were found in $[5,6,7,8,13],[4],[10],[15],[16],[1],[2]$ respectively. This paper deals with that problem for the class of finite supersoluble groups i.e. groups with an invariant series all of whose factors are cyclic. In addition, it goes further by describing exactly those values of $g$ for which the bound is attained. More precisely we prove:

TheOREM A. A supersoluble group of automorphisms of a compact Riemann surface of genus $g \geqslant 3$ has no more than $18(g-1)$ elements. A surface of genus $g=2$ can admit a supersoluble group of automorphisms of order 24.

THEOREM B: A necessary and sufficient condition for the existence of a Riemann surface of genus $g \geqslant 3$ that admits a supersoluble group of automorphisms of order $18(g-1)$ is that $3^{2}$ divides $g-1$ and the only other prime divisors of $g-1$ are congruent to $1 \bmod 3$.

2. Preliminaries. Groups of automorphisms of compact Riemann surfaces are quotient groups of Fuchsian groups. The basic results on Fuchsian groups for application to this problem are outlined in this section and can be found in [9]. A Fuchsian group is a discrete subgroup $\Gamma$ of orientation preserving isometries of the upper half plane $D$ with hyperbolic structure. If $D / \Gamma$ is a compact surface then $\Gamma$ has a presentation of the form

$$
\left(x_{1}, \ldots, x_{r}, a_{1}, b_{1}, \ldots, a_{g}, b_{g}: x_{i}^{m_{i}}(i=1, \ldots, r), \prod_{i=1}^{r} x_{i} \prod_{i=1}^{g} a_{i} b_{i} a_{i}^{-1} b_{i}^{-1}\right)
$$

and is said to have a signature

$$
\left(g ; m_{1}, \ldots, m_{r}\right)
$$

or to be a Fuchsian $\left(g ; m_{1}, \ldots, m_{r}\right)$-group. The integers $m_{1}, \ldots, m_{r}$ are called periods of $\Gamma$, and $g$ the genus.

Every Fuchsian group $\Gamma$ has an associated fundamental region whose hyperbolic area $\mu(\Gamma)$ depends only on the group. If $\Gamma$ has signature $(2.2)$ then

$$
\mu(\Gamma)=2 \pi\left[(2 g-2)+\sum_{i=1}^{r}\left(1-1 / m_{i}\right)\right]
$$

and in addition for a subgroup $\Gamma_{1}$ of finite index the following Riemann-Hurwitz index

Glasgow Math. J. 31 (1989) 321-327. 
formula holds:

$$
\mu\left(\Gamma_{1}\right) / \mu(\Gamma)=\left[\Gamma: \Gamma_{1}\right]
$$

It is well known that a compact Riemann surface of genus $g \geqslant 2$ can be represented as $D / \Gamma$, where $\Gamma$ is a Fuchsian group with signature $(g ;-)$ called a surface group of genus $g$. Moreover given a surface so represented, a finite group $G$ acts as a group of automorphisms if and only if there exists a Fuchsian group $\Lambda$ and a homomorphism $\theta$ from $\Lambda$ onto $G$ having $\Gamma$ as the kernel. Such a homomorphism is said to be a surface-kernel homomorphism and a group $G$ represented in such a form a surface-kernel factor group while $\Lambda$ is said to admit $G$ as a surface-kernel factor group.

It is also well known that a homomorphism from $\Lambda$ onto $G$ is a surface-kernel homomorphism if and only if it preserves the periods of $\Lambda$.

REMARK 2.1. We see that given a class of finite groups $\mathscr{F}$ the problem of finding the bound for $N(g, \mathscr{F})$ is equivalent to the problem of finding a Fuchsian group with minimal area that admits a group in $\mathscr{F}$ as a surface-kernel factor group.

This general method is standard (see e.g. [9]) and has been used to investigate this problem for the various classes of groups mentioned in the introduction.

3. On finite supersoluble groups generated by two elements of orders 2 and 3 respectively. We collect here some basic results on finite supersoluble groups which will be used later. Throughout $G^{\prime}$ denotes the commutator subgroup of $G$.

LEMMA 3.1. (a) If $G$ is a finite supersoluble group, then $G^{\prime}$ is nilpotent.

(b) If $H$ is a nilpotent normal subgroup of a finite group $G$ such that $G / H^{\prime}$ is supersoluble, then $G$ is also supersoluble.

(c) (Zappa) If $G$ is a finite supersoluble group then $G$ has an invariant series

$$
1=G_{0}<G_{1}<\ldots<G_{n}=G
$$

with cyclic factors of prime order of decreasing magnitude.

Proof. For (a) and (c) see [12, 5.4.8 and 5.4.10]. Result (b) is deducible from [12, 9.4.5].

Lemma 3.2. A group $G$ of order $2 \times 3^{n}$ generated by two elements of orders 2 and 3 is supersoluble.

Proof. We will prove the lemma by induction on $n$. Note that the lemma is true for $n=1$ and 2 since the only one of the five groups of order 18-the direct product of the cyclic group of order 3 and the dihedral group of order $6-$ can be generated in this way. Now assume that the assertion holds for all groups in question of order smaller than the order of $G$.

The Sylow 3-subgroup $G_{3}$ of $G$ is normal in $G$. Thus the generator of order 3 belongs to $G_{3}$ and if $G_{0}$ is the normal closure of that generator then $G_{0} \subseteq G_{3}$. Since however $G / G_{0}$ is either trivial or of order $2, G_{0}=G_{3}$. Now by using Reidemeister-Schreier algorithm for 
determining a presentation for subgroups of given groups, it is easy to see that $G_{0}$ is generated by two elements of order 3 . If $G_{0}$ is abelian then $n$ is either 1 or 2 . Assume then that $G_{0}$ is non-abelian. Then $G_{0}^{\prime}$ is non-trivial and hence $G / G_{0}^{\prime}$ is supersoluble by the induction hypothesis. Therefore $G$ is supersoluble by the previous lemma.

Leмma 3.3. Let $G$ be a supersoluble group of order $2 \times 3^{n}$ generated by two elements $x$ and $y$ of orders 2 and 3 respectively, whose product has order 18. Then $G_{a b} \cong Z_{6}$.

Proof. Let $G$ be the group in question and let $H$ be its normal subgroup of index 18 . First note that $y$ does not belong to $H$, otherwise $\tilde{G}=G / H$ would be the cyclic group of order 2 . Thus $\tilde{G}$ is a group of order 18 generated by two elements of orders 2 and 3 respectively. Thus, as noted in the proof of the previous lemma, $\tilde{G}_{a b} \cong Z_{6}$. So $G_{a b} \cong Z_{6}$ since $\tilde{G}_{a b}$ is its homomorphic image, whilst $G_{a b}$ is a homomorphic image of $\Gamma_{a b} \cong Z_{6}$, where $\Gamma$ is a Fuchsian group with signature $(0: 2,3,18)$ (see table below).

TABLE 4.1

\begin{tabular}{lccl}
\hline \multicolumn{1}{c}{$\Gamma$} & $\mu(\Gamma)$ & $\Gamma / \Gamma^{\prime}$ & \multicolumn{1}{c}{$\Gamma^{\prime}$} \\
\hline$(0 ; 2,3,7)$ & $\pi / 21$ & 1 & $(0 ; 2,3,7)$ \\
$(0 ; 2,3,8)$ & $\pi / 12$ & $Z_{2}$ & $(0 ; 3,3,4)$ \\
$(0 ; 2,3,9)$ & $\pi / 9$ & $Z_{3}$ & $(0 ; 2,2,2,3)$ \\
$(0 ; 2,3,10)$ & $2 \pi / 15$ & $Z_{2}$ & $(0 ; 3,3,5)$ \\
$(0 ; 2,3,11)$ & $5 \pi / 33$ & 1 & $(0 ; 2,3,11)$ \\
$(0 ; 2,3,12)$ & $\pi / 6$ & $Z_{6}$ & $(1 ; 2)$ \\
$(0 ; 2,3,13)$ & $7 \pi / 39$ & 1 & $(0 ; 2,3,13)$ \\
$(0 ; 2,3,14)$ & $4 \pi / 21$ & $Z_{2}$ & $(0 ; 3,3,7)$ \\
$(0 ; 2,3,15)$ & $\pi / 5$ & $Z_{3}$ & $(0 ; 2,2,2,5)$ \\
$(0 ; 2,3,16)$ & $5 \pi / 24$ & $Z_{2}$ & $(0 ; 3,3,8)$ \\
$(0 ; 2,3,17)$ & $11 \pi / 51$ & 1 & $(0 ; 2,3,17)$ \\
$(0 ; 2,3,18)$ & $2 \pi / 9$ & $Z_{6}$ & $(1 ; 3)$ \\
$(0 ; 2,4,5)$ & $\pi / 10$ & $Z_{2}$ & $(0 ; 5,5,2)$ \\
$(0 ; 2,4,6)$ & $\pi / 6$ & $Z_{2} \oplus Z_{2}$ & $(0 ; 2,2,3,3)$ \\
$(0 ; 2,4,7)$ & $3 \pi / 14$ & $Z_{2}$ & $(0 ; 7,7,2)$ \\
$(0 ; 2,5,5)$ & $\pi / 5$ & $Z_{5}$ & $(0 ; 2,2,2,2,2)$ \\
$(0 ; 3,3,4)$ & $\pi / 6$ & $Z_{3}$ & $(0 ; 4,4,4)$ \\
\hline
\end{tabular}

4. Supersoluble surface-kernel factors of Fuchsian groups with small area. Zomorrodian [15] has shown that a nilpotent automorphism group of a compact Riemann surface of genus $g \geqslant 2$ has no more than $16(g-1)$ elements. In terms of Fuchsian groups and its signatures (see Remark 2.1) this means that there is no Fuchsian group with area strictly smaller than $\pi / 4$ that admits a nilpotent surface-kernel factor. A finite nilpotent group is supersoluble $([12,5.4 .6])$. Thus in order to find a bound for the order of a supersoluble group of automorphisms of a compact Riemann surface we have to inspect the Fuchsian groups with area $\leqslant \pi / 4$ and find the one with minimal area that admits supersoluble surface-kernel factor groups.

By inspecting all Fuchsian groups with area $\leqslant 2 \pi / 9$ we are going to show that the ones that we are looking for are those with area $2 \pi / 9$ and $\pi / 6$, although the last one admits only one supersoluble surface-kernel factor group. To do that we need the 
above table of Fuchsian groups with area $\leqslant 2 \pi / 9$ as well as the signatures of their commutator subgroups.

The results given on p. 323 can, for example, be proved using a theorem of Singerman [14] and the Riemann-Hurwitz formula.

LemMa 4.1. Among the Fuchsian groups with area strictly smaller than $2 \pi / 9$ the group $\Gamma$ with signature $(0 ; 2,4,6)$ is the only one that admits a supersoluble surface-kernel factor group. Moreover $\Gamma$ admits only one such group (a semidirect product of the cyclic group of order 3 and the dihedral group of order 8).

Proof. The groups with signatures $(0 ; 2,3, k)$, where $k$ is relatively prime to 6 as perfect groups do not admit such factors.

Suppose that $\Gamma$ has a signature $(0 ; 2,3,8)$. Assume, by way of contradiction, that $G$ admits a supersoluble surface-kernel factor group $G$ and let $\theta$ be the corresponding surface-kernel homomorphism. $G^{\prime}$ is nilpotent by Lemma 3.1 and clearly is a surfacekernel factor group of $\Gamma^{\prime}$, where $\left.\theta\right|_{\Gamma^{\prime}}$, is the corresponding homomorphism. The group $\Gamma^{\prime}$ is generated by two elements $x_{1}, x_{2}$ subject to the relations $x_{1}^{3}=x_{2}^{3}=\left(x_{1} x_{2}\right)^{4}=1$. Since $G^{\prime}$ is a surface-kernel factor group of $\Gamma^{\prime}$ the homomorphism $\theta$ preserves the orders of $x_{1}, x_{2}$, $x_{1} x_{2}$. On the other hand since a finite nilpotent group is a direct product of its Sylow subgroups the order of $\theta\left(x_{1} x_{2}\right)$ is a power of 3 . A contradiction.

The groups with signatures $(0 ; 2,3,9),(0 ; 2,3,10)(0 ; 2,3,14), \quad(0 ; 2,3,15)$, $(0 ; 2,3,16),(0 ; 2,4,5)$, and $(0 ; 2,4,7)$ can similarly be ruled out. Thus it remains to consider the groups $(0 ; 2,3,12),(0 ; 2,3,18),(0 ; 2,4,6),(0 ; 2,5,5),(0 ; 3,3,4)$.

Let us consider the group $\Gamma$ with signature $(0 ; 2,3,12)$. Assume that $\Gamma$ admits a supersoluble surface-kernel factor group and let $G$ be one such group with minimal order. We will show first that the order of $G$ is of the form $2^{k} 3^{l}$. Assume, by way of contradiction, that $p \neq 3$ is the largest odd prime divisor of $G$. Then by the theorem of Zappa, $G$ possesses a normal subgroup $P$ of order $p$ and clearly $\Gamma$ admits $G / P$ as a surface-kernel factor group, which contradicts the minimality of $G$. Thus $|G|=2^{k} 3^{l}$. Again by the theorem of Zappa, $G$ possesses a normal subgroup $H$ of order 3 . Recall that the group $\Gamma$ is generated by two elements $x_{1}, x_{2}$ subject to the relations

$$
x_{1}^{2}=x_{2}^{3}=\left(x_{1} x_{2}\right)^{12}=1 .
$$

If the image of $x_{2}$ in $G$ belongs to $H$ then $G / H$ is the cyclic group of order 2 . Hence $|G|=6$, a contradiction. If the image of $\left(x_{1} x_{2}\right)^{4}$ in $G$ belongs to $H$ then $G / H$ is a group generated by two elements of orders 2 and 3 whose product has order 4 , i.e. is the symmetric group of degree 4 which fails to be supersoluble, a contradiction once more.

Neither the image of $x_{2}$ nor of $\left(x_{1} x_{2}\right)^{4}$ belongs to $H$. Consequently $\Gamma$ admits a group $G / H$ as a surface-kernel factor, which again contradicts the minimality of $G$. Thus the Fuchsian group with signature $(0 ; 2,3,12)$ does not admit supersoluble surface-kernel factor groups.

The groups with signatures $(0 ; 2,5,5)$ and $(0 ; 3,3,4)$ can be ruled out similarly.

Finally suppose that $\Gamma$ has the signature $(0,2,4,6)$ and let a group $G$ be its 
supersoluble surface-kernel factor group. Then $G^{\prime}$ is a nilpotent surface-kernel factor group of $(0 ; 2,2,3,3)$. Hence $G^{\prime}$ is generated by three elements $a, b, c$, that satisfy the following relations:

$$
a^{2}=b^{2}=c^{3}=(a b c)^{3}=1 .
$$

But since a finite nilpotent group is a product of its Sylow subgroups this set of relations implies that $a=b$. Hence $G^{\prime}$ is isomorphic to $Z_{6}$ and a supersoluble surface-kernel factor group of a $(0 ; 2,4,6)$-group has order 24 . One can readily check that the only such group is a semi-direct product of the cyclic group of order 3 and the dihedral group of order 8 .

In the next section we will show that the remaining Fuchsian group, the one with signature $(0 ; 2,3,18)$, admits infinitely many supersoluble surface-kernel factors.

5. Proofs of the announced results. We start with the proof of Theorem A.

The second part follows directly from the proof of Lemma 4.1. So let $G$ be a supersoluble group of automorphisms of a compact Riemann surface of genus $g \geqslant 3$. Then $G=\Lambda / \Gamma$, where $\Lambda$ is a Fuchsian group and $\Gamma$ is the surface group of genus $g$.

By the Lemma $4.1 \Lambda$ is either a Fuchsian group with signature $(0 ; 2,4,6)$ or $\mu(\Lambda) \geqslant 2 \pi / 9$.

If $\Lambda$ were a Fuchsian group with signature $(0 ; 2,4,6)$ then on the one hand $|G|=24$ by Lemma 4.1 while on the other $|G| \geqslant 48$ by the Riemann-Hurwitz index formula (2.4), a contradiction. Therefore $\mu(\Lambda) \geqslant 2 \pi / 9$ and consequently $|G|=\mu(\Gamma) / \mu(\Lambda)$ $\leqslant 4 \pi(g-1) /(2 \pi / 9)=18(g-1)$.

Proof of Theorem B. First we will prove the necessity of the conditions in question. Let $G$ be a supersoluble group of automorphisms of a compact Riemann surface of genus $g \geqslant 3$, of order $18(g-1)$.

By Remark 2.1, $G$ is a surface-kernel factor of the $(0 ; 2,3,18)$-group since this is the only Fuchsian group with the area $2 \pi / 9$. Thus $G$ is a group generated by two elements $x$ and $y$ of order 2 and 3 respectively whose product has order 18.

First we will show that $g-1$ is odd. Assume, by way of contradiction, that 2 divides $g-1$. By the theorem of Zappa there is a normal subgroup $H$ of $G$ such that $\tilde{G}=G / H$ is a 2 -group of order $\geqslant 4$. On the other hand the generator $y$ of $G$ becomes the identity in $\tilde{G}$, so $\tilde{G}$ has order $\leqslant 2$, a contradiction.

Let $p \neq 3$ divide $g-1$. By using Lemma 3.3, the fact that $G^{\prime}$ is a direct product of its Sylow $p$-subgroups each of which is normal in $G$, and then the theorem of Zappa, we can deduce that there is a supersoluble surface-kernel factor group $\bar{G}$ of $(0 ; 2,3,18)$, of order $2 \times 3^{n} p$. From the proof of Lemma 3.2 it follows that $\tilde{G}$ contains a subgroup $\tilde{G}_{0}$ of index 2 generated by two elements of order 3 .

If $p \not \equiv 1 \bmod 3$ then a Sylow 3 -subgroup $\tilde{H}$ of $\tilde{G}_{0}$ is normal and thus on the one hand $\tilde{G}_{0} / \tilde{H}$ is a group of order $p$ whilst on the other it is generated by two elements of order 3 , a contradiction.

Now by using the theorem of Zappa once more we deduce that $G$ has a factor group $\tilde{G}$ of order $2 \times 3^{n}$, which is also a supersoluble surface-kernel factor of $(0 ; 2,3,18)$. By 
Lemma $3.3 \tilde{G}$ contains $\tilde{G}^{\prime}$ as a subgroup of index 6 . Moreover $\tilde{G}^{\prime}$ is generated by two elements whose commutator has order 3 . Thus $\tilde{G}^{\prime}$ is non abelian and in particular $3^{3}$ divides $\left|\tilde{G}^{\prime}\right|$. Thus $3^{2}$ divides $g-1$.

In the case where $g-1$ is a power of 3 , the sufficiency of the conditions given in Theorem B follows from recent work of May [11] on $M^{*}$-groups, which are maximal groups of automorphisms of compact bordered Klein surfaces. Such groups are quotients of the group of hyperbolic isometries generated by reflections in the sides of a triangle with angles $\pi / 2, \pi / 3, \pi / q[3]$. The integer $q$ is called an index of the group, and in [11] it is shown that there exist supersoluble $M^{*}$-groups of index 18 and order $4.3^{n+2}$ for all $n \geqslant 2$. The algebraic genus $g$ of the corresponding Klein surface is given by $12(g-1)=$ $4.3^{n+2}$ and it has $\frac{1}{3}(g-1)$ boundary components. The subgroup of index two in the $M^{*}$-group which is the quotient of the $(0 ; 2,3,18)$-group acts on a compact Riemann surface of the same topological genus as that of the Klein surface [3] and so we obtain a supersoluble group of automorphisms of a compact Riemann surface of genus $3^{n}+1$.

Let $D$ be an integer such that the prime divisors of $D$ are all congruent to $1 \bmod 3$, and let $H=\langle h\rangle$ be a cyclic group of order $D$. Then the congruence $\alpha^{2}-\alpha+1=0$ $(\bmod D)$ has a solution and $\phi(h)=h^{\alpha}$ is an automorphism of $H$ of order 6. From above, given $n \geqslant 2$, there exists a supersoluble group $G$ of automorphisms of order $18(g-1)$ of a compact Riemann surface of genus $g$ with $g=3^{n}+1$. Thus $G$ has generators $x_{1}, x_{2}$ satisfying $x_{1}^{2}=x_{2}^{3}=\left(x_{1} x_{2}\right)^{18}=1$. By the Lemma $3.3 G / G^{\prime} \cong Z_{6}$ and so there is a homomorphism $T: G \rightarrow\langle\phi\rangle \subseteq \operatorname{Aut}(H)$, with the images of the generators $x_{1}, x_{2}$ of order 2 and 3 respectively being $\phi^{3}$ and $\phi^{2}$.

We form the semidirect product $\tilde{G}$ of $H$ by $G$ with respect to the homomorphism $T$ so that the multiplication in $\tilde{G}$ is defined by

$$
\left(h_{1}, f_{1}\right)\left(h_{2}, f_{2}\right)=\left(h_{1}\left(T\left(f_{1}\right)\left(h_{2}\right)\right), f_{1} f_{2}\right), \quad h_{i} \in H, f_{i} \in G .
$$

Since $H$ is normal in $\tilde{G}$ and cyclic, $\tilde{G} / H \cong G$ is supersoluble, it follows that $\tilde{G}$ is supersoluble.

The elements $\left(1, x_{1}\right)$ and $\left(h, x_{2}\right)$ in $\tilde{G}$ have orders 2 and 3 respectively and their product has order 18 . Also the commutator

$$
\left[\left(1, x_{1}\right),\left(h, x_{2}\right)\right]=\left(h^{-2},\left[x_{1}, x_{2}\right]\right) .
$$

Now $\left[x_{1}, x_{2}\right] \in G^{\prime}$ which acts trivially on $H$ and has order a power of 3 . Thus a suitable power of the commutator above is equal to $(h, 1)$. It follows that $\left(1, x_{1}\right),\left(h, x_{2}\right)$ generate $\tilde{G}$ and exhibit $\tilde{G}$ as a surface-kernel factor group of the $(0 ; 2,3,18)$-group. This completes the proof of Theorem $B$.

Acknowledgement. This paper was written when the first author was visiting the Departamento de Matematicas Fundamentales of the Universidad a Distancia in Madrid, while in receipt of a Spanish Government postdoctoral grant. He would like to thank the members of the department, particularly its director Prof E. Bujalance, and J. M. R. Sanjurjo of Complutense University, for their hospitality. 


\section{REFERENCES}

1. B. P. Chetiya, Groups of Automorphisms of compact Riemann surfaces, Ph.D. thesis, Birmingham University.

2. B. P. Chetiya and K. Patra, On metabelian groups of automorphisms of compact Riemann surfaces, J. London Math. Soc. 33 (1986), 467-472.

3. N. Greenleaf and C. L. May, Bordered Klein surfaces with maximal symmetry. Trans. Amer. Math. Soc. 274 (1982) 265-283.

4. W. J. Harvey, Cyclic groups of automorphisms of a compact Riemann surface, Quart. J. Math. Oxford Ser. 217 (1966), 86-98.

5. A. Hurwitz, Uber algebraische Gebilde mit eindeutigen Transformationen in sich, Math. Ann. 41 (1893), 403-442.

6. J. Lehner and $M$. Newman, On Riemann surfaces with maximal automorphism groups, Glasgow Math. J. 8 (1967), 102-112.

7. A. M. Macbeath, On a theorem of Hurwitz, Proc. Glasgow Math. Assoc, 5 (1961), 90-96.

8. A. M. Macbeath, Generators of the linear fractional groups, Proc. Symp. Pure Math. Vol. XII, Amer. Math. Soc. (1969), 14-32.

9. A. M. Macbeath, Discontinuous groups and birational transformations, Proc. Dundee Summer School 1961.

10. C. Maclachlan, Abelian groups of automorphisms of compact Riemann surfaces, Proc. London Math. Soc. (3) 15 (1965), 699-712.

11. C. L. May, Supersoluble $M^{*}$-groups, Glasgow Math. J. 30 (1988), 31-40.

12. D. J. S. Robinson, A course in the Theory of Groups (Springer Verlag, 1982).

13. D. Singerman, Automorphisms of compact non-orientable Riemann surfaces, Glasgow Math. J., 12 (1971), 50-59.

14. D. Singerman, Subgroups of Fuchsian groups and finite permutation groups. Bull. London Math. Soc. 2 (1970), 319-323.

15. R. Zomorrodian, Nilpotent automorphism groups of Riemann surfaces, Trans. Amer. Math. Soc. 288 (1985), 241-255.

16. R. Zomorrodian, Classification of $p$-groups of automorphisms of Riemann surfaces and their lower series, Glasgow Math. J. 29 (1987), 237-244.

\author{
InSTYtut Matematyki WSP \\ Chodkiewicza 30 \\ 85-064 Bydgoszcz, Poland. \\ AND \\ Universidad a Distancia \\ Depto de Matem, Fund. \\ 28040 MADRID, SPAIN.
}

Department of Mathematics

UNIVERSITY OF ABERDEEN

The Edward Wright Building

Dunbar STREeT

AberdeEn AB9 2TY 